\section{Selbst ist der Mann? Ungewöhnliche Maßnahme bei akuter Meatusstenose}

\author{
Juckende Beschwerden mit scheinbar eingetrocknetem urethralen \\ Ausfluss wurden hier vorschnell unter "Geschlechtskrankheit" verbucht. \\ In seiner Not griff der Patient zum Messer.
}

Ein in 33-jähriger beschnittener Mann - hatte vor einigen Tagen eine Nothilfe aufgesucht, weil er im Bereich des äußeren Harnröhrenmeatus einen heftigen Juckreiz verspürte und Probleme beim Wasserlassen hatte. Unter der Verdachtsdiagnose einer sexuell übertragbaren Infektion wurde er mit einem Rezept für Doxycyclin wieder nach Hause geschickt.

Nachdem er den ganzen Tag über kein Wasser lassen konnte, suchte er noch einmal die Nothilfe auf. Diesmal verordnete man wegen des Verdachts auf eine Gonorrhoe Amoxicillin und riet dem Mann, eine Spezialambulanz für Urologie aufzusuchen.

Die Beschwerden nahmen zu. Der Harndrang wurde unerträglich, sodass der Mann nun die Dinge in die eigene Hand nahm und einen weißlichen fleischartigen Lappen über dem Meatus mit einem scharfen Mes- ser entfernte. Daraufhin konnte er wenigstens wieder Wasser lassen.

In der urologischen Spezialambulanz stellte man die Diagnose eines progressiven Lichen sclerosus im Bereich des Harnröhrenmeatus, der zu einer Meatusstenose geführt hatte. Die weichen, weißlich verfärbten und lederartig erschei-

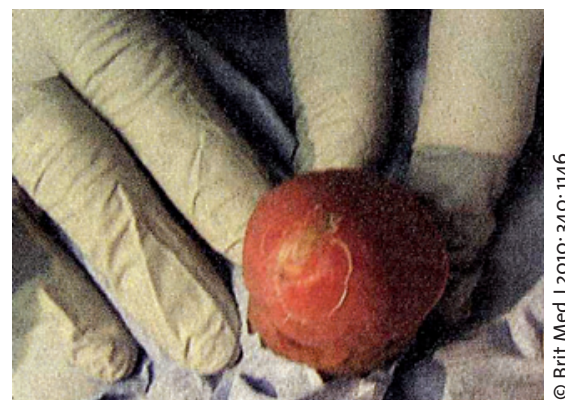

Meatusstenose nach Selbstbehandlung und vor der Op nenden Veränderungen des Lichen sclerosus werden von Nichtexperten oft für eingetrockneten uretralen Ausfluss gehalten. Dadurch wird die Notwendigkeit einer Dilatation des Meatus verkannt.

Beim Lichen sclerosus handelt es sich um eine Präkanzerose, die in etwa 5\% der Fälle tatsächlich in ein Malignom übergeht. Unter Verwendung von steroidhaltigen Salben kann der Prozess allerdings zum Stillstand gebracht werden.

Fazit: Hautveränderungen im Bereich des männlichen Genitales werden wahrscheinlich zu häufig und reflexartig mit sexuell übertragbaren Erkrankungen in Verbindung gebracht. Es wäre interessant, die näheren Umstände der Vorstellung, das Aussehen und Verhalten des Patienten und den Ablauf des Anamnesegesprächs zu erfahren, da hier möglicherweise die Verdachtsdiagnose in eine falsche Richtung lief. Eine genaue Analyse des Harnstrahls hätte z.B. bei dieser seit längerer Zeit bestehenden Veränderung auf die richtige Spur geführt.

H. S. FüeßI

Winceslaus SJ, Wijesurendra C.

Brit Med J 2010; 340: 1146

\title{
Bier als Risikofaktor für Psoriasis
}

Eine Assoziation zwischen Alkoholkonsum und Entstehung bzw. Verschlechterung einer bestehenden Schuppenflechte wird seit langem vermutet. Allerdings wurden die Angaben zu den Alkoholmengen in den FallKontroll-Studien meist retrospektiv gemacht. Was die Trinkgewohnheiten von Frauen angeht, wurde bislang keine signifikante Beziehung gefunden.

$\mathrm{n}$ dieser Untersuchung nutzte man $\mathrm{Da}-$ ten der Nurses' Health Study II: fast 83.000 Frauen zwischen 27 und 44 Jahren, die initial keine Psoriasis hatten. Die Teilnehmerinnen waren zwischen 1991 und 2005 regelmäßig hinsichtlich Menge und Art der konsumierten alkoholhaltigen Getränke befragt worden. Bei 1.069 entwickelte sich in dieser Zeit eine Psoriasis.

Dabei erwies sich Bier als einziges alkoholisches Getränk, welches das PsoriasisRisiko signifikant erhöhte: Für fünf oder mehr Biere in der Woche (1 Drink war definiert als 12,8 g Alkohol) errechnete sich ein 1,8faches Psoriasis-Risiko im Vergleich zu Frauen, die nur Alkoholfreies tranken. In Fällen, in denen die Diagnose mittels eines spezifischen Fragebogens zusätzlich bestätigt wurde, war das Risiko sogar mehr als verdoppelt (2,3fach), auch nach Berücksichtigung anderer Risikofaktoren wie Alter, Rauchen, BMI etc.

Nachdem weder der Genuss von Wein noch von Spirituosen mit der Entwicklung einer Psoriasis korrelierte, handelt es sich bei dem unbekannten Risikofaktor offenbar um eine Nicht-Alkohol-Komponente des Biers. Möglicherweise, spekulieren die Autoren, spielt die Gerste eine Rolle, die beim Brauen als Stärkequelle dient und im Endprodukt verbleibt: Wie viele backfähige Getreidearten auch enthält sie das
Kleber-Eiweiß Gluten. Für diese Proteine wird seit einiger Zeit eine Assoziation zur Psoriasis beschrieben. So hat man bei $\mathrm{Pa}$ tienten häufiger als in der Allgemeinbevölkerung erhöhte Titer an Anti-GliadinAntikörpern nachgewiesen. Außerdem konnte man in solchen Fällen durch eine glutenfreie Kost günstige Effekte auf den Hautzustand erzielen.

Dass alkoholfreies Bier die Entwicklung einer Psoriasis nicht begünstigte, erklärt man sich damit, dass für dessen Herstellung weniger Getreide verwendet werde.

Fazit: Bei diesen prospektiv erhobenen Daten zeigte sich, dass neben der Menge vor allem auch die Art der konsumierten Alkoholika entscheidend ist. Frauen, die ein erhöhtes Psoriasis-Risiko haben, sollten möglichst wenig Bier trinken. wpa

Qureshi AA et al. Alcohol intake and risk of incident psoriasis in US women. Arch Dermatol 2010; Epub ahead of print. 